\title{
Dietary nitrate supplementation effect on dynamic cerebral autoregulation in normoxia and acute hypoxia
}

Article

Accepted Version

Horiuchi, G., Rossetti, G. M. K. ORCID: https://orcid.org/00000002-9610-6066 and Oliver, S. J. (2022) Dietary nitrate supplementation effect on dynamic cerebral autoregulation in normoxia and acute hypoxia. Journal of Cerebral Blood Flow \& Metabolism, 42 (3). pp. 486-494. ISSN 1559-7016 doi: https://doi.org/10.1177/0271678X20910053 Available at https://centaur.reading.ac.uk/97685/

It is advisable to refer to the publisher's version if you intend to cite from the work. See Guidance on citing.

To link to this article DOI: http://dx.doi.org/10.1177/0271678X20910053

Publisher: SAGE Publications

All outputs in CentAUR are protected by Intellectual Property Rights law, including copyright law. Copyright and IPR is retained by the creators or other copyright holders. Terms and conditions for use of this material are defined in the End User Agreement. 


\section{CentAUR}

Central Archive at the University of Reading

Reading's research outputs online 
Title: Dietary nitrate supplementation effect on dynamic cerebral autoregulation in normoxia and acute hypoxia

Running title: Dietary nitrate and cerebral autoregulation

Masahiro Horiuchi ${ }^{1}$, Gabriella M.K. Rossetti ${ }^{2}$, Samuel J. Oliver ${ }^{2}$

1. Division of Human Environmental Science, Mt. Fuji Research Institute

2. Extremes Research Group, College of Human Sciences, Bangor University

Corresponding author: Masahiro Horiuchi, Ph.D

Address; Kami-yoshida 5597-1, Fuji-yoshida city, Yamanashi, 4030005, Japan

e-mail; mhoriuchi@mfri.pref.yamanashi.jp

TEL; +81-555-72-6198

FAX; +81-555-72-6216 


\begin{abstract}
We tested the hypothesis that increasing the nitric oxide (NO) bioavailability by dietary nitrate would recover the hypoxic-induced reduction in dynamic CA. Twelve healthy males (age $21 \pm 2$ years) completed four days of dietary supplementation with a placebo or inorganic nitrate drink (140-ml beetroot juice) followed by 60 -min of normoxia or hypoxia (fraction of inspired oxygen $\left[\mathrm{FiO}_{2}\right]=13 \%$ ). Duplex ultrasonography was used to perform volumetric change-based assessment of dynamic CA in the internal carotid artery (ICA). Dynamic CA was assessed by rate of regulation (RoR) of vascular conductance using the thigh-cuff method. Four days of beetroot supplementation increased circulating nitrate by $208[171,245] \mu \mathrm{M}$ (mean difference [95\% confidence interval]) compared with placebo. Dynamic CA was lower in hypoxia than normoxia (RoR $\Delta-0.085[-0.116,-0.054]$ ). Compared with placebo, nitrate did not alter dynamic CA in normoxia $(\operatorname{RoR} \Delta-0.022[-0.060,0.016])$ or hypoxia $(\operatorname{RoR} \Delta 0.017[-0.019$, 0.053]). Further, nitrate did not affect ICA vessel diameter, blood velocity or flow in either normoxia or hypoxia. Increased bioavailability of NO through dietary nitrate supplementation did not recover the hypoxia-induced reduction in dynamic CA. This suggests the mechanism of hypoxia-induced reduction in dynamic CA does not relate to the availability of NO.
\end{abstract}


Key words: nitric oxide, acute hypotension, cerebral blood flow, vasodilation, vascular conductance 


\section{INTRODUCTION}

Cerebral autoregulation (CA) is essential to protect the brain against ischaemic injury, capillary damage, and edema ${ }^{1,2}$. Dynamic CA is the maintenance of cerebral blood flow $(\mathrm{CBF})$ at constant levels despite acute changes in cerebral perfusion pressure or in systemic blood pressure ${ }^{3}$. The physiological mechanisms responsible for the control of dynamic cerebral autoregulation are thought to involve a combination of nitric oxide (NO), neurogenic (sympathetic and cholinergic) and myogenic (stretching vascular smooth muscle) factors ${ }^{1}$. While dynamic CA normally ensures the preservation of CBF, including to transient hypertension ${ }^{4,5}$, studies demonstrate that dynamic CA is reduced during transient hypotension in normoxic and hypoxic conditions (i.e. acute hypoxia and at high altitude $)^{4-14}$. The impairment in dynamic CA is characterized by a blunted increase in cerebrovascular conductance (CVC) in response to systemic hypotension, preventing normalization of $\mathrm{CBF}^{13}$. The mechanism by which hypoxia reduces dynamic CA is unclear but may relate to nitric oxide (NO) availability ${ }^{15}$.

$\mathrm{NO}$ is a potent vasodilator and plays a vital role in the regulation of $\mathrm{CVC}$, and consequently $\mathrm{CBF}^{16}$. Previous research in humans, breathing normal ambient air, has shown dynamic CA to be substantially reduced when the enzyme NO synthase is inhibited $^{15}$. In oxygen-depleted environments, the L-arginine pathway, which is helped by the enzyme NO-synthase, has a decreased ability to generate $\mathrm{NO}^{17}$. NO can also be 
generated by the serial reduction of nitrate $\left(\mathrm{NO}_{3}^{-}\right)$to nitrite $\left(\mathrm{NO}_{2}^{-}\right)$and then $\mathrm{NO}$, providing an alternative pathway for NO production in hypoxia ${ }^{18,19}$. Dietary nitrate supplementation has been shown to be effective to increase the NO bioavailability ${ }^{20}$, and improve cerebral regulation and CBF in normoxia ${ }^{21,22}$. Specifically, dietary nitrate supplementation has previously been shown to enhance cerebral perfusion ${ }^{21}$ and increase middle cerebral artery blood flow velocity via a reduction in cerebrovascular resistance $^{22}$. In addition, dietary nitrate has been shown to reverse the hypoxia-induced impairment in peripheral NO-dependent endothelial function ${ }^{23}$. Combined, this suggests dietary nitrate may be effective to reverse the hypoxia-induced impairment in dynamic CA.

The aim of this study was to determine the role of NO availability in the hypoxiainduced reduction of dynamic CA. We hypothesized nitrate supplementation would reverse the hypoxia-induced reduction in dynamic $\mathrm{CA}$, which would provide evidence that NO plays a central role in the mechanism of hypoxia-induced reduction in dynamic CA.

\section{METHODS}

Participants 
All procedures were approved by the ethical committee of Mt. Fuji Reseach Institute and were performed in accordance with the guidelines of the Declaration of Helsinki (ECMFRI-01-2014). After a detailed explanation of all study procedures, including the possible risks and benefits of participation, each participant gave his written consent. Twelve healthy male participants with a mean age of $21 \pm 2$ years, $174 \pm 5 \mathrm{~cm}$, and body mass $69 \pm 8 \mathrm{~kg}$ (mean \pm standard deviation [SD]) were enrolled. They were free from any cardiovascular or cerebrovascular diseases, and were not taking any medications. Participants engaged in regular recreational sports (1-2 h per day, 2-4 days per week). None of the participants was exposed to an altitude higher than $1500 \mathrm{~m}$ within 6 months before the study. In addition, participants were asked to abstain from caffeine for $12 \mathrm{~h}$ and from strenous exercise and alcohol for at least $24 \mathrm{~h}$ before the study. Participants were familiarized with measurement techniques (i.e., thigh-cuff testing for dynamic CA and measurement of blood flow in the intrnal carotid artery [ICA]). All studies were performed in an environmental chamber (TBR-4, 5SA2GX, Tabai Espec Co, Ltd., Tokyo, Japan) with set at an ambient temperature of $24^{\circ} \mathrm{C}$ and at relative himidity of $40 \%$.

\section{Study design}

The study followed a double-blinded placebo-controlled crossover design. All participants performed two trials, each assessing dynamic CA in both normoxia and 
hypoxia after four days of (1) nitrate-rich beetroot juice (nitrate) and (2) nitrate-depleted beetroot juice (placebo) dietary supplementation. These two trials were performed with at least a 2-week washout period and in random order. An overview of the study protocol is shown in Figure 1.

\section{Supplementation}

For the three days before the trials, participants consumed $140 \mathrm{ml}$ per day of $\mathrm{NO}_{3}{ }^{-}$-rich beetroot juice (nitrate; Beet It, James White Drinks, Ltd., Ipswich, UK) or $140 \mathrm{~mL}$ per day of $\mathrm{NO}_{3}{ }^{-}$-depleted beetroot concentrate (placebo; James White Drinks, Ltd., Ipswich, UK). Both participants and researchers were blinded to the drink contents until the completion of the study. Participants were also provided a list of foods rich in $\mathrm{NO}_{3}{ }^{-}$, and instructed to avoid the consumption of these foods and to otherwise maintain their normal dietary intake for the duration of the study. In addition, they were asked to abstain from the use of antibacterial mouthwash for the duration of the study, as it eliminates the oral bacteria that reduce $\mathrm{NO}_{3}{ }^{-}$to $\mathrm{NO}_{2}{ }^{-24}$.

\section{Study procedures}

On each study day (nitrate or placebo), participants consumed their final dose of nitrate or placebo on arrival to the laboratory, about $1.5 \mathrm{~h}$ before the first dynamic CA test. After a 30 min supine and 45 min semi-recumbent rest on a manually inclined bed 
(A4524-0096L, Paramount Bed Co., Ltd., Tokyo, Japan), a venous blood sample (10 $\mathrm{mL}$ ) was collected from the antecubital vein into a vacutainer and immediately centrifuged at $1000 \mathrm{~g}$ for $15 \mathrm{~min}$ at $4^{\circ} \mathrm{C}$ (Kubota 5200, Kubota Co., Ltd., Tokyo, Japan) to separate serum from whole blood. The serum samples were frozen at $-80^{\circ} \mathrm{C}$ for further analysis of $\mathrm{NO}_{3}{ }^{-}$content by SRL Co., Ltd. (Tokyo, Japan). Briefly, after deproteinizaation, serum $\mathrm{NO}_{3}{ }^{-}$was measured by using high-performance liquid chromatography ${ }^{25}$. After attachment of all devices, normoxic baseline values were measured for $5 \mathrm{~min}$; then, dynamic CA assessment in normoxia was performed. Thereafter, hypoxic gas $\left(\mathrm{FiO}_{2}\right.$ 0.13) was supplied via a commercial tent (about $5000 \mathrm{~L}$ ) with a hypoxic gas generating system (Hypoxico Everest Summit 2: Will Co., Ltd., Tokyo, Japan). Hypoxic baseline values were measured during the last 5 min of the 60 min resting hypoxic exposure. Next, dynamic CA assessment in hypoxia was performed. Inspired oxygen concentration was verified before and after each experiment using a metabolic cart (AE-310s; Minato Medical Science, Osaka, Japan).

\section{Dynamic CA assessmemt}

Dynamic CA was assessed with participants sat on a comfortable chair in a semirecumbent position. After 2 min of baseline data in normoxia were recorded, bilateral thigh-cuffs (width $22 \mathrm{~cm}$ ) were inflated to a pressure of $220 \mathrm{mmHg}$ for $3 \mathrm{~min}$ using a custom cuff inflator. To evaluate dynamic CA, the cuff was then rapidly deflated, 
causing a transient drop in arterial blood pressure, and measurements were continued for an additional minute. Participants were instructed to remain relaxed and were not given feedback regarding the elasped time during cuff occlusion. This protocol was repeated two or three times at 5-min intervals to allow for recovery to the resting value $^{26}$. If the first or second trial reduction in mean arterial pressure (MAP) was $<15$ $\mathrm{mmHg}^{27}$, a third trial was performed, and the average value of two trials was used ${ }^{13}$.

\section{Measurements}

Right ICA measurements were performed $1.0-1.5 \mathrm{~cm}$ distal to the carotid bifurcation with a Doppler ultrasound set at 10.0 MHz and a linear transducer (Logic-e; GE Healthcare, Tokyo, Japan). For baseline ICA flow measurement, the ICA blood flow was averaged over 2 min during the last 5 min of the initial 30-min resting exposure. For the dynamic ICA blood flow measurements, blood flow was continuously measured during the thigh cuff test. To calculate the average ICA blood flow, we analyzed the mean vessel diameter $\left(D_{\text {mean }}\right)$ and flow velocity as described in a previous study ${ }^{28}$. Briefly, after obtaining a clear image of the vessel using the brightness mode, the mean vessel diameter was calculated as: mean diameter $=($ systolic diameter $\times 1 / 3)+$ (diastolic diameter $\times 2 / 3$ ). The time-averaged mean flow velocity obained using the pulse wave mode was defined as the mean blood flow velocity $\left(\mathrm{V}_{\text {mean }}\right.$; in centimeters per second). Blood flow was calculated by multiplying the cross-sectional area $\times 60$ (in 
milliliters per minute). Throughout the measurement, care was taken to ensure that the probe position was stable, the insonation angle did not vary $\left(<60^{\circ}\right.$ in all cases $)$ and the sample volume was positioned in the center of the vessel and adjusted to cover the width of the vessel diameter. Heart rate (HR) was monitored using three-lead ECG. Pulmonary ventilation $\left(\dot{\mathrm{V}}_{\mathrm{E}}\right)$ and partial pressure of end-tidal carbon dioxide output $\left(\mathrm{P}_{\mathrm{ETCO}}\right)$ were measured by a breath-by-breath gas analyzer (AE-310S; Minato Medical Science, Osaka, Japan). Standard gases $\left(\mathrm{O}_{2} 15.23 \%, \mathrm{CO}_{2} 4.999 \%\right.$, and $\mathrm{N}_{2}$ balance $)$ and room air were used to calibrate the gas analyzer before each test. Beat-by-beat MAP was measured using finger photoplethysmography from the middle or index finger of the left hand (MUB-101; Medi-sens, Saiatama, Japan). Peripheral arterial oxygen saturation $\left(\mathrm{SpO}_{2}\right)$ was monitored by finger pulse oximetry (PULFIS WB-100, Japan Precision Instruments Inc., Gunma, Japan).

\section{Data analysis}

We calculated the rate of regulation $(\mathrm{RoR})$ as an indicator of dynamic $\mathrm{CA}^{29}$. For calculation of RoR, baseline values for MAP, ICA blood flow and cerebrovascular conductance (ICA/MAP) were defined as the mean during the $4 \mathrm{~s}$ immediately before the thigh-cuff release. The RoR was calculated from the slope of the regression line between ICA conductance and the time from 1.0 to $4.0 \mathrm{~s}$ after thigh-cuff deflation and normalized to the degree of cuff-release-induced hypotension. The RoR $=(\Delta \mathrm{ICA}$ 
conductance/ $\Delta \mathrm{t}) / \Delta \mathrm{MAP}$, where $\Delta \mathrm{ICA}$ conductance/ $\Delta \mathrm{t}$ is the slope of the linear regression between ICA conductance and time $(\mathrm{t})$, and $\triangle \mathrm{MAP}$ is the magnitude of the blood pressure step, which was calculated by subtracting the baseline MAP from the MAP averaged during the interval from 1.0 to $4.0 \mathrm{~s}$. In a previous study, the data from 1.0 to $3.5 \mathrm{~s}$ after cuff deflation were used to calculate dynamic $\mathrm{CA}^{29}$; however, we extended this time to $4.0 \mathrm{~s}$ after cuff deflation to ensure beat-by-beat blood flow data for at least three cardiac cycles ${ }^{13,28}$. Trials in which the reduction in MAP was $<15 \mathrm{mmHg}$ were excluded from analysis, and the average of two trials was used. The coefficient of variation of this measure is $13 \%$.

\section{Statistics}

Values are expressed as means \pm SD unless otherwise stated. Two-way repeatedmeasures ANOVAs (oxygen $\times$ supplement) were used for comparisons of cardiorespiratory, hemodynamic and dynamic CA variables across conditions. P values $<0.05$ were considered to indicate statistical significance. The magnitude of effect of nitrate supplementation on the hypoxia-induced decline in dynamic CA variables was determined by ANCOVA comparison of nitrate and placebo hypoxia trials (with normoxia placebo as the covariate), and interpreted in relation to a priori meaningful differences ${ }^{30}$. A sample size estimation for the primary analysis indicated that 10 participants were needed to produce an $80 \%$ chance of obtaining statistical significance 
at the 0.05 level for a two-tailed design, based on a minimum important difference of 0.076 RoR, a standard deviation of the difference of 0.05 , and an estimated correlation of $0.4^{11}$. Statistical analyses were performed using commercial software packages (Sigma Stat 3.5, Hulinks, Chicago, IL, USA; SPSS V25, IBM Corp, Armonk; NY).

\section{RESULTS}

Serum nitrate

Four days inorganic nitrate supplementation increased serum $\mathrm{NO}_{3}{ }^{-}$concentrations compared to placebo (mean difference [95\% confidence interval]: $\Delta 208[171,245] \mu \mathrm{M}$; $P<0.001)$.

\section{Cardiorespiratory and hemodynamic responses at rest}

Effect of hypoxia. Compared to normoxia, acute hypoxia decreased $\mathrm{SpO}_{2}$, and increased HR, ICA diameter, ICA blood flow, and CVC (all $P<0.05$; Table 1). Further, acute hypoxia tended to increase $\dot{\mathrm{V}}_{\mathrm{E}}(P=0.06)$ and decrease $\mathrm{P}_{\mathrm{ETCO}}(P=0.09)$. Hypoxia did not alter MAP or ICA blood flow velocity at rest $(P>0.05$; Table 1$)$.

Effect of nitrate. Dietary nitrate supplementation did not affect any of the resting cardiovascular or cerebrovascular responses in either normoxia or hypoxia (all $P>0.05$; Table 1). 


\section{Dynamic cerebral autoregulation}

Effect of hypoxia. Thigh-cuff release elicited similar MAP reductions in hypoxia and normoxia ( $\mathrm{P}=0.7$; Table 2$)$. In contrast, the ICA blood flow nadir in response to thighcuff was greater in hypoxia than normoxia $(P<0.001$; Table 2$)$. Dynamic CA assessment (1-4 s after deflation) indicated that hypoxia did not alter the $\triangle \mathrm{MAP}$, but did decrease the slope of CVC $(P<0.05$; Table 2). Consequently, RoR was decreased in hypoxia compared to normoxia $(\Delta-0.085[-0.116,-0.054] ; P<0.05$; Figure 3$)$.

Effect of nitrate. Thigh-cuff release elicited similar reductions in MAP and ICA flow after nitrate and placebo in normoxia and hypoxia (Table 2 ; interactions $P>0.9$ ). During dynamic CA assessment (1-4s after deflation), nitrate had no effect on any dynamic CA variable in normoxia or hypoxia when compared to the placebo (Table 2, Figures 2 and 3). Specifically, compared to placebo, nitrate supplementation did not alter RoR in normoxia $(\Delta-0.022[-0.060,0.016] ; P=0.2)$ or hypoxia $(\Delta 0.017[-0.019$, 0.053]; $P=0.3$ ). Further, compared to placebo, nitrate supplementation did not effect the hypoxia-induced decline in dynamic CA variables (Figure 2): MAP $(\Delta-0.06[-3.62$, 3.50]; $P=0.9)$, ICA flow $(\Delta-1[-26,23] ; P=0.9), \operatorname{CVC}(\Delta-0.003[-0.009,0.002] ; P=$ $0.2)$, or $\operatorname{RoR}(\Delta 0.020[-0.010,0.051] ; P=0.2$

\section{DISCUSSION}


This study is the first to investigate the effects of dietary nitrate supplementation on dynamic CA in hypoxia. The major findings of this study are therefore that increased bioavailability of NO by dietary nitrate supplementation did not influence dynamic CA in acute hypoxia or recover the hypoxia-induced reduction in dynamic CA (Figure 3). In addition, we also show that dietary nitrate supplementation did not influence cerebrovascular responses, including dynamic CA, whilst participants breathed normal ambient air (normoxia). A particular strength of this study was the method used to determine dynamic CA, i.e. duplex Doppler ultrasound in conjunction with the traditional thigh-cuff test. In brief, we used duplex Doppler ultrasound as it enables the assessment of blood flow velocity and vessel diameter, which contrasts the easier-toperform and more commonly used TCD method that only assesses blood flow velocity. Previous research has shown the thigh-cuff method, which imposes a large and abrupt change in blood pressure, is more sensitive to interventions, such as hypoxia, than transfer function analysis that examines spontaneous fluctuations in blood pressure ${ }^{26}$. The importance of using these methods is emphasised when considering the only other study to have examined dietary nitrate supplementation on dynamic CA in normoxia reported inconclusive effects ${ }^{31}$ that may have resulted from using TCD in conjunction transfer function analysis, which is a less sensitive method to assess dynamic CA than used in the present study. 
In our study, acute hypoxia reduced dynamic CA which is a consistent finding with many other studies $^{6-14}$. Indeed, 10 of 12 participants had reduced dynamic CA with hypoxia irrespective of supplementation. However, in contrast with our hypothesis, and previous research that suggests NO may have an important role in regulating brain vascular tone ${ }^{32}$ and dynamic $\mathrm{CA}^{15}$, dietary nitrate supplementation did not influence any physiological determinant of dynamic CA (Figure 2), or dynamic CA (Figure 3), differently than the placebo in acute hypoxia. Indeed, the mean difference in RoR between nitrate and placebo and the $95 \%$ confidence intervals indicate any effect of nitrate is trivial in magnitude. This finding suggests NO bioavailability has limited influence on the hypoxia-induced reduction in dynamic $\mathrm{CA}$ observed in young healthy adults. Of note, our results do not discount the role of $\mathrm{NO}$ in $\mathrm{CBF}$ responses including dynamic $\mathrm{CA}$ in hypoxia. $\mathrm{CBF}$ regulation is a complex, multifaceted physiological process, of which the action of NO is a key component ${ }^{33}$. The data from this study simply suggest that insufficient bioavailability of NO is not the primary cause of hypoxia-induced reduction in dynamic CA.

A possible alternative mechanism for the hypoxia-induced reduction in dynamic CA could be attributed to increased vasodilation at rest ${ }^{29}$. Hypoxia is a potent vasodilator $^{34}$, and therefore cerebral blood vessels may already be close to maximally dilated in hypoxia, limiting capacity for further vasodilation to maintain CVC during dynamic CA. In our study hypoxia substantially increased ICA diameter at rest (Table 
1), which may have limited the capacity for further dilation in dynamic CA response to the thigh cuff release. Regions with already elevated CBF at rest in hypoxia have been previously shown to minimally increase $\mathrm{CBF}$ with experimental manipulations known to cause vasodilation ${ }^{35}$, supporting the idea that baseline diameter influences capacity for further dilation. Indeed, previous research supports that dynamic CA depends on basal vascular tone, and that the autoregulatory response in middle cerebral artery blood flow velocity is slower when cerebral vessels are already dilated ${ }^{29}$. Similarly, lower cerebrovascular resistance (higher cerebrovascular conductance) resulted in higher transfer function gain, indicating that dynamic $\mathrm{CA}$ is reduced when cerebral vessels are dilated $^{36}$. Other factors, including prostaglandins ${ }^{37-40}$, and sympathetic nerve activity $^{33,41}$, have been shown to contribute the regulation of CBF and may also be responsible for the hypoxia-induced reduction in dynamic CA.

\section{Limitations and perspectives}

Our findings should be considered carefully within the context they were obtained. To assess dynamic CA we measured changes in ICA blood vessel diameter and blood velocity flow. Global CBF however consists of $\sim 75 \%$ ICA flow and $\sim 25 \%$ vertebral artery (VA) flow ${ }^{42}$ so, although technically challenging, a more complete assessment of CA would have included both ICA and VA. Further, as one previous magnetic resonance imaging study observed regional but not global CBF differences after nitrate 
supplementation $^{21}$, future studies examining the influence of dietary nitrate on regional extra- and intracranial cerebral blood flow and autoregulation are warranted. Dietary nitrate supplementation has been shown to be effective to recover peripheral vascular function at high altitude ${ }^{23}$. In contrast, dietary nitrate did not affect cerebral vascular function during hypoxic exposure in the present study. This heterogenous response between vascular beds is consistent with a previous study where dietary nitrate increased muscle oxygenation in hypoxia, but did not affect cerebral oxygenation ${ }^{43}$. In combination these findings provide evidence dietary nitrate and NO influence peripheral and cerebral vascular responses to hypoxia by different mechanisms. The limited effects of dietary nitrate observed on dynamic CA in this study may be a consequence of the young healthy population recruited. Indeed, as other studies in young healthy individuals we report no effect of dietary nitrate on resting physiological responses in normoxia or hypoxia ${ }^{23,44,45}$. Whether dietary nitrate influences cerebrovascular responses and dynamic CA in persons with reduced vascular function, poor NO bioavailability, or clinical populations with cerebrovascular disease remains to be investigated. Future research should also resolve whether reductions in dynamic CA are related to an increase in adverse outcomes such as headache and Acute Mountain Sickness, which are common in hypoxic conditions ${ }^{7,9,13,46}$. Resolving this is important as dynamic CA could be a useful screening-tool to identify those persons at risk of hypoxia-induced adverse events. 


\section{CONCLUSION}

In conclusion, a four-day dietary inorganic nitrate supplementation did not affect resting cerebrovascular responses or dynamic CA in either normoxia or hypoxia (after 60-min exposure with $13 \% \mathrm{O}_{2}$ ) in healthy men. This suggests $\mathrm{NO}$ availability is not responsible for hypoxia-induced reduction in dynamic CA. 
Acknowledgements: The authors thank all participants for their time and effort. The authors also thank Mrs. Kirihara-Handa Yoko, and Hamada-Endo Junko for their technical assistance.

Funding: This study was partly supported by the funding of Japan Society for the Promotion of Science (\# 26440268 to M.H.)

Author contributions: M. H. designed and performed the study. M.H., G. M. K. R., and S. J. O. analysed and interpreted results. M.H., G. M. K. R., and S. J. O. prepared tables and figures. M.H. drafted the first manuscript. M.H., G. M. K. R., and S. J. O. edited and revised the manuscript. All authors have approved the final version.

Conflict of interest: The authors declared no conflicts of the interest with respect to this study. 


\section{References}

1. Tzeng YC, Ainslie PN. Blood pressure regulation IX: Cerebral autoregulation under blood pressure challenges. Eur J Appl Physiol 2014; 114: 545-559.

2. Paulson OB, Strandgaard S, Edvinsson L. Cerebral autoregulation. Cerebrovasc Brain Metab Rev 1990; 2: 161-192.

3. Lassen NA. Cerebral blood flow and oxygen consumption in man. Physiol Rev 1959; 39: 183-238.

4. Aaslid R, Blaha M, Sviri G, et al. Asymmetric dynamic cerebral autoregulatory response to cyclic stimuli. Stroke 2007; 38(5):1465-9.

5. Tzeng YC, Willie CK, Atkinson G, et al. Cerebrovascular regulation during transient hypotension and hypertension in humans. Hypertension 2010; 56: 268273.

6. Iwasaki KI, Ogawa Y, Shibata S, et al. Acute exposure to normobaric mild hypoxia alters dynamic relationships between blood pressure and cerebral blood flow at very low frequency. J Cereb Blood Flow Metab 2007; 27: 776-784.

7. Bailey DM, Evans KA, James PE, et al. Altered free radical metabolism in acute mountain sickness: implications for dynamic cerebral autoregulation and bloodbrain barrier function. J Physiol 2009; 587: 73-85.

8. Subudhi AW, Panerai RB, Roach RC. Acute hypoxia impairs dynamic cerebral autoregulation: results from two independent techniques. J Appl Physiol 2009; 
107: 1165-1171.

9. Subudhi AW, Panerai RB, Roach RC. Effects of hypobaric hypoxia on cerebral autoregulation. Stroke 2010; 41: 641-646.

10. Nishimura N, Iwasaki K, Ogawa Y, et al. Decreased steady-state cerebral blood flow velocity and altered dynamic cerebral autoregulation during 5-h sustained 15\% O2 hypoxia. J Appl Physiol 2010; 108: 1154-1161.

11. Ogoh S, Nakahara H, Ainslie PN, et al. The effect of oxygen on dynamic cerebral autoregulation: critical role of hypocapnia. J Appl Physiol 2010; 108: 538-543.

12. Ainslie PN, Lucas SJE, Fan J-L, et al. Influence of sympathoexcitation at high altitude on cerebrovascular function and ventilatory control in humans. $J$ Appl Physiol 2012; 113: 1058-1067.

13. Horiuchi M, Endo J, Dobashi S, et al. Effect of progressive normobaric hypoxia on dynamic cerebral autoregulation. Exp Physiol 2016; 82: 1496-1514.

14. Subudhi AW, Fan JL, Evero O, et al. AltitudeOmics: Effect of ascent and acclimatization to $5260 \mathrm{~m}$ on regional cerebral oxygen delivery. Exp Physiol 2014; 99: 772-781.

15. White RP, Vallance P, Markus HS. Effect of inhibition of nitric oxide synthase on dynamic cerebral autoregulation in humans. Clin Sci 2000; 99: 555-60.

16. Toda N, Ayajiki K, Okamura T. Cerebral blood flow regulation by nitric oxide: recent advances. Pharmacol Rev 2009; 61: 62-97. 
17. McQuillan LP, Leung GK, Marsden PA, et al. Hypoxia inhibits expression of eNOS via transcriptional and posttranscriptional mechanisms. Am J Physiol Circ Physiol 2017; 268: 1-1.

18. Duncan C, Dougall H, Johnston P, et al. Chemical generation of nitric oxide in the mouth from the enterosalivary circulation of dietary nitrate. Nat Med 1995; 1: 546551.

19. Lundberg JO, Weitzberg E, Gladwin MT. The nitrate-nitrite-nitric oxide pathway in physiology and therapeutics. Nat Rev Drug Discov 2008; 7: 156-167.

20. Wylie LJ, Kelly J, Bailey SJ, et al. Beetroot juice and exercise: pharmacodynamic and dose-response relationships. J Appl Physiol 2013; 115: 325-336.

21. Presley TD, Morgan AR, Bechtold E, et al. Acute effect of a high nitrate diet on brain perfusion in older adults. Nirtic Oxide 2011; 24: 34-42.

22. Bond V, Curry BH, Adams RG, et al. Effects of dietary nitrates on systemic and cerebrovascular hemodynamics. Cardiol Res Pract 2013: 435629, DOI: $10.1155 / 2013 / 435629$.

23. Bakker E, Engan H, Patrician A, et al. Acute dietary nitrate supplementation improves arterial endothelial function at high altitude: a double-blinded randomized controlled cross over study. Nitric Oxide 2015; 50: 58-64.

24. Govoni M, Jansson EA, Weitzberg E, et al. The increase in plasma nitrite after a dietary nitrate load is markedly attenuated by an antibacterial mouthwash. Nitric 
Oxide 2008; 19: 333-337.

25. Takahashi H, Nakanishi T, Nishimura M, et al. Measurements of serum levels of nitrate ions in men and women: implications of endothelium-derived relaxing factor in blood pressure regulation and atherosclerosis. Journal Cardiovasc Pharmacol 992; 20: S214-S216.

26. Subudhi AW, Grajzel K, Langolf RJ, et al. Cerebral autoregulation index at high altitude assessed by thigh-cuff and transfer function analysis techniques. Exp Physiol 2015; 100: 173-181.

27. Tiecks FP, Lam AM, Aalsid R, et al. Comparison of static and dynamic cerebral autoregulation measurements. Stroke 1995; 26: 1014-1019.

28. Sato K, Fisher JP, Seifert T, et al. Blood flow in internal carotid and vertebral arteries during orthostatic stress. Exp Physiol 2012; 97: 1272-1280.

29. Aaslid R, Lindegaard KF, Sorteberg W, et al. Cerebral autoregulation dynamics in humans. Stroke 1989; 20: 45-52.

30. Hopkins WG, Batterham AM. Error rates, decisive outcomes and publication bias with several inferential methods. Sport Med 2016; 46: 1563-1573.

31. Fan J-L, O’Donnell T, Gray CL, et al. Dietary nitrate supplementation enhances cerebrovascular CO2 reactivity in a sex-specific manner. J Appl Physiol 2019; 127: $760-769$.

32. Van Mil AHM, Spilt A, Van Buchem MA, et al. Nitric oxide mediates hypoxia- 
induced cerebral vasodilation in humans. J Appl Physiol 2002; 92: 962-966.

33. Ainslie PN, Ogoh S. Regulation of cerebral blood flow in mammals during chronic hypoxia: a matter of balance. Exp Physiol 2010; 95: 251-262.

34. Ainslie PN, Subudhi AW. Cerebral blood flow at high altitude. High Alt Med Biol 2014; 15: 133-40.

35. Lawley JS, Macdonald JH, Oliver SJ, et al. Unexpected reductions in regional cerebral perfusion during prolonged hypoxia. J Physiol 2017; 595: 935-947.

36. Serrador JM, Sorond FA, Vyas M, et al. Cerebral pressure-flow relations in hypertensive elderly humans: transfer gain in different frequency domains. $J$ Appl Physiol 2005; 98: 151-159.

37. Fan JL, Burgess KR, Thomas KN, et al. Influence of indomethacin on the ventilatory and cerebrovascular responsiveness to hypoxia. Eur J Appl Physiol 2011; 111: 601-610.

38. Harrell JW, Schrage WG. Cyclooxygenase-derived vasoconstriction restrains hypoxia-mediated cerebral vasodilation in young adults with metabolic syndrome. Am J Physiol Circ Physiol 2014; 306: H261-H269.

39. Bowton DL, Haddon WS, Prough DS, et al. Theophylline effect on the cerebral blood flow response to hypoxemia. Chest 1988; 94: 371-375.

40. Meno JR, Ngai AC, Richard Winn H. Changes in pial arteriolar diameter and CSF adenosine concentrations during hypoxia. J Cereb Blood Flow Metab 1993; 13 : 
214-220.

41. Curran-Everett DC, Meredith MP, Krasney JA. Acclimatization to hypoxia alters cerebral convective and diffusive O2 delivery. Respir Physiol 1992; 88: 355-371.

42. Schöning M, Walter J, Scheel P. Estimation of cerebral blood flow through color duplex sonography of the carotid and vertebral arteries in healthy adults. Stroke 1994; 25: 17-22.

43. Masschelein E, Van Thienen R, Wang X, et al. Dietary nitrate improves muscle but not cerebral oxygenation status during exercise in hypoxia. J Appl Physiol 2012; 113: 736-745.

44. Lefferts WK, Hughes WE, White CN, et al. Effect of acute nitrate supplementation on neurovascular coupling and cognitive performance in hypoxia. Appl Physiol Nutr Metab 2016; 4: 133-141.

45. Rossetti GMK, Macdonald JH, Wylie LJ, et al. Dietary nitrate supplementation increases acute mountain sickness severity and sense of effort during hypoxic exercise. J Appl Physiol 2017; 123: 983-992.

46. Cochand NJ, Wild M, Brugniaux JV, et al., Sea-level assessment of dynamic cerebral autoregulation predicts susceptibility to acute mountain sickness at high altitude. Stroke 2011; 42(12):3628-30. 


\section{Figure Legends}

Figure 1. The protocol of the present study. BL, baseline; dCA, dynamic cerebral autoregulation assessment; $\mathrm{FiO}_{2}$, fraction of inspired oxygen.

Figure 2. Comparisons in the components of dynamic cerebral autoregulation among the four conditions. The supplement contrast is defined by dashed (placebo) versus solid (nitrate) lines, the oxygen contrast is defined by black (normoxia) versus grey (hypoxia) lines. As such, the four conditions are represented as follows: placebo normoxia, dashed black line; nitrate normoxia, solid black line; placebo hypoxia, dashed grey line; nitrate hypoxia, solid grey line. All values are presented as changes from baseline to cuff release, normalised to baseline in each condition. Hypoxia (A) had no effect on mean arterial pressure (MAP), (B) decreased internal carotid artery (ICA) flow, and (C) decreased cerebrovascular conductance (CVC) following thigh cuff release $\left({ }^{*} P<0.05\right)$. However, nitrate had no effect on (A) MAP, (B) ICA flow, or (C) CVC (all $P>0.05)$. Values are means \pm SD. $* P<0.05$ between normoxia and hypoxia.

Figure 3. Dynamic cerebral autoregulation (rate of regulation: RoR) in normoxia and hypoxia after placebo and nitrate supplementation. * Significant difference between normoxia and hypoxia $(\mathrm{P}>0.05)$. Values are means \pm standard deviation (SD). 